\title{
New insights into the nature of mid-infrared emission associated with massive star formation: disks and outflow
}

\author{
James M. De Buizer \\ Gemini Observatory, Casilla 603, La Serena, Chile \\ email: jdebuizer@gemini.edu
}

\begin{abstract}
Recent observations in the mid-infrared $(5-25 \mu \mathrm{m})$ of massive young stellar sources have yielded a surprising result: many show evidence of mid-infrared emission from outflows and jets. These observations correlate well with other larger-scale outflow indicators and their geometries, such as what is seen in shock-excited $\mathrm{H}_{2}$ and $\mathrm{CO}$ emission. In some cases these mid-infrared observations identify the local maser emission as outflow or jet related.
\end{abstract}

Keywords. ISM: jets and outflows, infrared: ISM, circumstellar matter, stars: early-type

\section{Introduction}

Thanks to the increase of facility-class mid-infrared imagers on large aperture telescopes $(8-10 \mathrm{~m})$, we are achieving high spatial resolutions in the mid-infrared $(\sim 0.25$ 0.60") that allow us to see the detailed morphologies of the mid-infrared environments of massive young stellar sources. Mid-infrared (MIR) emission is usually attributed to circumstellar disk dust emission, however recent observations are showing circumstellar structure that is interpreted as arising from the directly heated dust on the walls of outflow cavities or from material caught up in outflow. An outflow cavity can be created by a molecular outflow or jet punching a hole in the dense clump of obscuring material surrounding a young stellar source. Direct associations of outflows and jets with individual massive stars are few. Outflows from massive stars may demonstrate that they form by accretion processes similar to low mass stars.

There have been two recent publications showing evidence of MIR emission from jets and outflows from massive young stellar objects. The clearest example comes from the observations of G35.20-0.74 by De Buizer (2006a), which show MIR emission from the jet cavity previously detected at other wavelengths. The larger-scale MIR structure of NGC 7538 IRS 1 observed by De Buizer \& Minier (2005) also shows what is believed to be emission from an outflow. The third and newest addition to this list is IRAS $20126+4104$. Using Michelle on Gemini North, high spatial resolution images revealed that the MIR emission at the center of this region is distributed in a double-lobed structure similar to the $2 \mu \mathrm{m}$ emission (Sridharan et al. 2005). De Buizer (2006b) argues that the MIR, near-IR, and maser emission are likely coming from the outflow cavities centered on the central radio continuum source at the heart of this region.

\section{References}

De Buizer, J. M. 2006a, ApJ 642, L57

De Buizer, J. M. 2006b, ApJ submitted

De Buizer, J. M. \& Minier, V. 2005, ApJ 628, L151

Sridharan, T. K., Williams, S. J. \& Fuller, G. A. 2005, ApJ 631, L73 